\title{
Structure-Borne Noise at PWM Excitation Using an Extended Field Reconstruction Method and Modal Decomposition
}

\author{
Janez Luznar ${ }^{1}$ - Janko Slavič ${ }^{2}{ }^{*}$ - Miha Boltežar² \\ 1Domel, d.o.o., Slovenia \\ 2University of Ljubljana, Faculty of Mechanical Engineering, Slovenia
}

\begin{abstract}
Pulse-width modulation (PWM) represents a carrier-frequency-dependent structural excitation. The PWM's excitation harmonics are also reflected in the air gap's electromagnetic forces, the vibration response and the resulting structure-borne noise. The last of these can be numerically predicted with a multiphysics finite element analysis (FEA) containing electronic, electromagnetic, mechanical and acoustic field problems. The multiphysics FEA are precise, but computationally inefficient and consequently inadequate for parametric studies. This paper introduces a method for a fast structure-borne noise prediction at PWM excitation. The presented approach contains the Extended field reconstruction method (EFRM) to handle the magnetic saturation and slotting effects in magnetics, and the modal decomposition to couple the electromagnetic and mechanical domains. Finally, the structure-borne sound power level is calculated via the vibration-velocity response. Indeed, this approach demands a pre-calculation of the basis functions and modal parameters from the FEA, but afterwards the effect of the different PWM excitation cases can be evaluated in a few seconds. The proposed method can calculate the structure-borne noise at PWM excitation accurately and is more than $10^{4}$ times faster than the conventional multiphysics FEA approach.
\end{abstract}

Keywords: carrier frequency, electromagnetic forces, extended field reconstruction method, modal decomposition, structure-borne noise

Highlights

- $\quad$ The structure-borne noise of PWM controlled machines can be reduced by the appropriate carrier-frequency selection in accordance with the structural dynamics.

- Based on the extended field reconstruction method, a fast method for structure-borne noise prediction at PWM excitation is introduced.

- $\quad$ The proposed method was shown to calculate the structure-borne noise at custom PWM excitation accurately and efficiently.

- $\quad$ Parametric study with densely spaced PWM carrier-frequency show a $30 \mathrm{~dB}(\mathrm{~A})$ difference in total sound power level.

\section{INTRODUCTION}

In permanent-magnet synchronous motor (PMSM) the variable speed can be controlled by pulsewidth modulation (PWM), which composes current waveforms of the desired fundamental frequency component together with a number of higher switching harmonics [1]. The latter enriches the Maxwell force spectrum, escalating the vibrations and the noise of electromagnetic origin [2]. Electromagnetic noise depends on the different motor types, motor powers, rotor speeds, PWM techniques and the carrier frequency [3]. The influence of the PWM carrier frequency on the structure-borne noise was experimentally researched in [4], where densely spaced carrier frequency measurements indicated a strong variation in the total emitted noise. However, the influence of the PWM carrier frequency on the structure-borne noise can also be estimated numerically, as shown in this article.

An accurate assessment of the noise in electrical machines requires a multiphysics analysis encompasing electronic, electromagnetic, mechanical and acoustic field problems, which can be solved by using an analytical or a numerical approach. Analytical methods allow quick computation, but suffer from poor accuracy and are usually limited to simple geometries [5] to [7]. In contrast, a finite element analysis (FEA) can obtain accurate results, but requires substantial computational resources [8] to [10]. The most time-consuming part in this multiphysics analysis is the electromagnetic transient simulation, which can be replaced with the computationally efficient field reconstruction method (FRM).

The FRM utilizes the field generated by one stator slot and a permanent magnet (PM) over one pole to construct the entire field distribution in the air gap [11]. Therefore, by using the FRM, the magnetic flux density for an arbitrary stator current excitation can be reconstructed efficiently. Sutthiphornsombat et al. [12] used the FRM to compute the electromagnetic forces and employed an optimization method to minimize the force pulsation and consequently reduce the acoustic noise. However, the coupling between the electromagnetic and the structural model was not involved. Furthermore, Torregrossa et al. [13] proposed an efficient computational model for a fast 
and accurate calculation of the electromagnetically induced vibrations in a PMSM using the FRM and knowledge of the mechanical impulse response. The vibration response was calculated with a convolution of the excitation force and the mechanical impulse response, which could be better handled directly in the frequency domain by using a frequency-response matrix.

The listed investigations show the great usefulness of the FRM, which reduces the computation time compared to the FEA, but is only applicable within the linear magnetic region of ferromagnetic materials [11]. To consider the magnetic saturation and slotting effects, an extended field reconstruction method (EFRM) was recently introduced by Gu et al. [14], where the field density distribution in the air gap can be reconstructed by using a set of pre-calculated basis functions. Comparisons with the FEA showed that the EFRM has an acceptable accuracy and takes significantly less time to compute [14]. However, there is a lack of vibro-acoustic investigations using the EFRM, which could expand the usage of previously investigated FRM-based methods.

The aim of this investigation is to establish a fast multiphysics numerical modeling of the structure-borne noise at PWM excitation. The proposed method is programmed with our own code and includes FEA imports, necessary for the EFRM and the structural model calculations. The manuscript is organized as follows. Section 1 presents the conventional method to simulate the structure-borne noise at PWM excitation with the multiphysics FEA. Section 2 presents the steps of the proposed fast multiphysics numerical simulation method, based on the EFRM and the modal decomposition. Section 3 shows the case study and the validation of the proposed method with the FEA. Section 4 shows the parametric study at different PWM excitations to emphasize the computational efficiency. Section 5 draws the conclusions.

\section{SIMULATION USING FINITE ELEMENT ANALYSES}

The conventional method to simulate structure-borne noise at PWM excitation, which is used later to compare the results to the newly proposed approach, is made with a multiphysics FEA containing all these domains:

- Electronic model: controlling the power electronics to generate a 3-phase PWM voltage excitation.

- Electrical model: the voltage excitation will cause electrical currents in the 3-phase windings, inducing spatially distributed electromagnetic flux waves in the air-gap of the motor.

- Electromagnetic model: the induced rotating flux density waves will cause both the tangential and radial force components generating the motor torque and unwanted vibrations.

- Structural model: the response of the structure depends on the frequency harmonics of the electromagnetic forces and the corresponding structural behavior of the motor.

- Acoustic model: surface vibrations will cause the pressure variations in the surrounding air, leading to radiated acoustic noise.

Different models can be weakly coupled since there is no significant feedback from the mechanical domain to the electromagnetic domain, and also none from the acoustic domain to the mechanical domain [15]. The case study presented in Section 3 is modelled with the commercial software package ANSYS 18.1 using the following modules:

\subsection{Simplorer and Maxwell}

A co-simulation with Maxwell and Simplorer is used to link the electromagnetic model with the electrical circuit for a 3-phase voltage-source inverter, as shown in Fig. 1. Transient simulation at PWM voltage excitation demands a small time step $(\leq 1 \mu \mathrm{s})$ to obtain the well-discretized PWM voltage pulses and, therefore, the appropriate frequency domain of the electromagnetic forces. To analyze the machine in a steady state, a transient simulation must accomplish at least a few electric cycles (at least 3 for the case study in Section 3). The electromagnetic forces acting on the tips of the stator's teeth are calculated within the last simulation cycle and transformed in the frequency domain for further analyses.

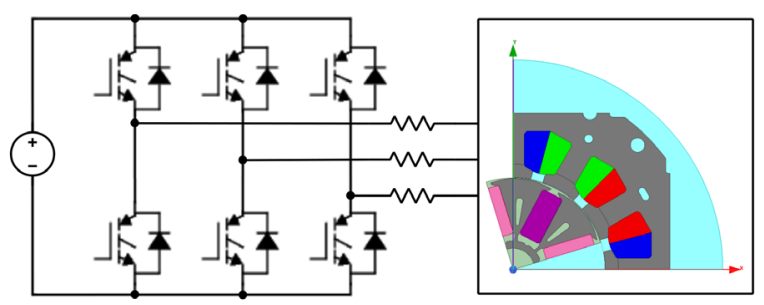

Fig. 1. Co-simulation using Maxwell and Simplorer in ANSYS 18.1

\subsection{Modal and Harmonic Response}

The Modal analysis and Harmonic response modules are used to couple the electromagnetic forces and the structural model, resulting in vibrational and 
acoustic responses, as shown in Fig. 2. The Modal analysis of the 3D mechanical model is used to obtain the structural dynamics parameters, i.e., the mode shapes, mode frequencies and mode damping, which represent the basis for further response calculations. By importing the modal results and the excitation magnetic forces, the vibration-velocity response is calculated with the Harmonic response module using the mode-superposition method. Finally, the vibration-velocity response of all the external surfaces is imported into another Harmonic Response module to simulate the acoustics and to calculate the sound power level of the structure-borne noise.

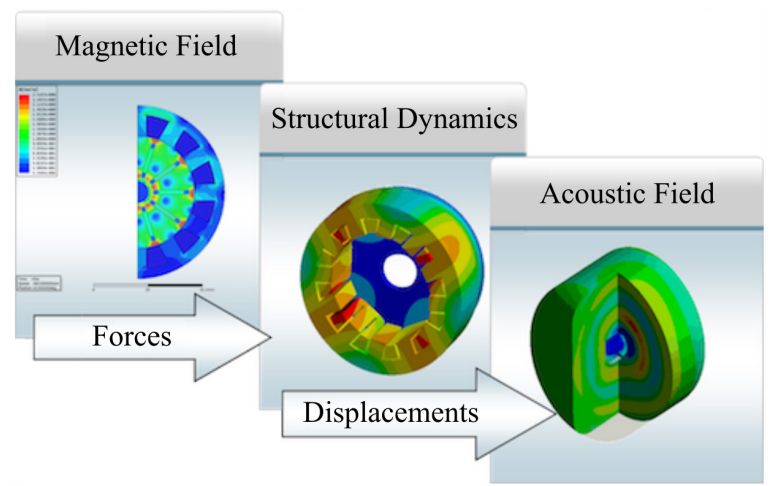

Fig. 2. Using the modal analysis and Harmonic response modules to calculate the vibration response and simulate acoustics in ANSYS 18.1

\section{FAST MULTIPHYSICS SIMULATION METHOD, BASED ON EFRM AND MODAL DECOMPOSITION}

As the conventional FEA multiphysics simulation is time consuming, an efficient multiphysics method, combining the EFRM and modal decomposition, is proposed in this investigation. The EFRM has been used to model the electric and electromagnetic domains, while the vibration velocity is calculated with the mechanical response model, defined by the modal decomposition. This approach requires some data pre-calculation from the FEA, but then the structure-borne noise at custom PWM excitation can be predicted efficiently.

\subsection{Custom PWM Excitation}

The PWM time domain presents a sequence of positive and negative voltage pulses, which result in a broadband frequency excitation. The program code to generate the PWM phase excitations $\left(u_{a}, u_{b}, u_{c}\right)$ for different PWM parameters, i.e., carrier type, carrier frequency, fundamental frequency and amplitude, was developed from scratch [4]. The PWM excitation frequency contents involve the fundamental component $f_{1}$ with additional switching harmonics at the frequencies $f_{h}$ [16]:

$$
f_{h}=n \cdot f_{c} \pm k \cdot f_{1},
$$

where $n=1,2,3, \ldots$, and $f_{c}$ is the carrier frequency. When $n$ is odd, $k= \pm 2, \pm 4, \ldots$, and when $n$ is even, $k= \pm 1, \pm 5, \ldots$ Fig. 3 shows the voltage harmonics for sine-triangle PWM with the fundamental at $100 \mathrm{~Hz}$ and the carrier frequency at $3000 \mathrm{~Hz}$.

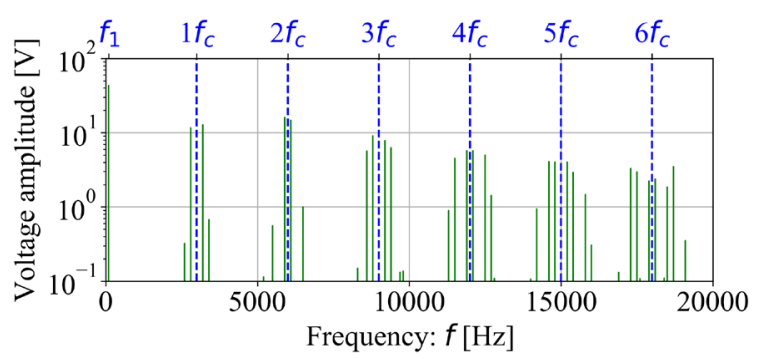

Fig. 3. Frequency contents for a sine-triangle PWM

\subsection{Electric Model of the PMSM}

In order to implement the EFRM, the 3-phase quantities (voltages, currents and flux linkages) are transformed into the $d q$ rotor reference frame, as shown for the voltages in Eq. (2) using the transformation matrix $T$, defined in Eq. (3):

$$
\begin{gathered}
{\left[\begin{array}{l}
u_{d} \\
u_{q}
\end{array}\right]=T \cdot\left[\begin{array}{l}
u_{a} \\
u_{b} \\
u_{c}
\end{array}\right],} \\
T=\frac{2}{3}\left[\begin{array}{ccc}
\cos (\theta) & \cos \left(\theta-\frac{2 \pi}{3}\right) & \cos \left(\theta+\frac{2 \pi}{3}\right) \\
-\sin (\theta) & -\sin \left(\theta-\frac{2 \pi}{3}\right) & -\sin \left(\theta+\frac{2 \pi}{3}\right)
\end{array}\right],
\end{gathered}
$$

where $u_{d}, u_{q}$ are the $d$ and $q$ components of the stator voltage vector, $\theta$ is the angle between the stator fixed $a$ axis and the rotor rotating $d$ axis. The vector representation of the transformation is presented in Fig. 4, where $\omega_{e}$ is the synchronous electrical speed. The $a$ axis points towards the center of the flux linkage $\mathrm{A}$, the $d$ axis is pointed at the center of the $\mathrm{PM}$, and the $q$ axis is defined as being 90 electrical degrees ahead of the $d$ axis. 


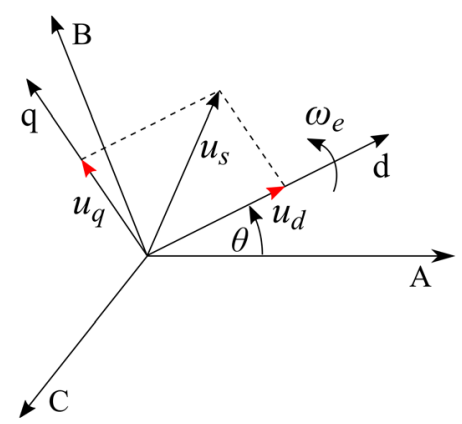

Fig. 4. Vector representation of the $a b c$ to $d q$ frame transformation

The voltage equations of the PMSM in the rotor reference $d q$-axis frame are defined in Eq. (4) [17]:

$\left[\begin{array}{l}u_{d} \\ u_{q}\end{array}\right]=\left[\begin{array}{cc}R_{s}+p L_{d} & -\omega_{e} L_{q}+p L_{d q} \\ \omega_{e} L_{d}+p L_{q d} & R_{s}+p L_{q}\end{array}\right]\left[\begin{array}{l}i_{d} \\ i_{q}\end{array}\right]+\left[\begin{array}{c}0 \\ \omega_{e} \psi_{m}\end{array}\right]$

where $i_{d}, i_{q}, L_{d}, L_{q}, L_{d q}, L_{q d}$ are the $d$ and $q$ axis currents, self and mutual inductances, respectively. $R_{S}$ is the phase resistance, $\psi_{m}$ is the flux linkage due to the permanent magnets and $p=d / d t$. Like in [18], we used the field components in the air gap to estimate the flux linkages, which can be calculated by integrating the radial flux density $B_{n}$ under each stator phase $i$ [18] and [19]:

$$
\psi_{i}=P N \int_{0}^{2 \pi / P} B_{n} L_{s k} r \mathrm{~d} \theta, \quad i=a, b, c,
$$

where $P$ represents the number of magnetic pole pairs, $N$ is the number of conductors in each coil, $L_{s k}$ is the stack length and $r$ is the radius of the closed contour in the air-gap. Furthermore, the values of $\psi_{d}$ and $\psi_{q}$ can be defined using the transformation matrix, Eq. (3). The incremental self and mutual inductances can be then determined according to Eq. (6):

$$
\begin{aligned}
& L_{d}=\partial \psi_{d} / \partial i_{d}, \quad L_{d q}=\partial \psi_{d} / \partial i_{q}, \\
& L_{q d}=\partial \psi_{q} / \partial i_{d}, \quad L_{q}=\partial \psi_{q} / \partial i_{q} .
\end{aligned}
$$

As shown in Section 2.3, the magnetic flux density can be reconstructed using the EFRM. Therefore, the flux linkages and inductances can also be calculated with Eqs. (3), (5) and (6). Lastly the custom PWM voltage excitation causes the transient currents $i_{d}$ and $i_{q}$, which can be numerically calculated step-bystep using the electrical model, Eq. (4) together with magnetic coupling, which is included via the EFRM based flux linkages and inductances.

\subsection{Magnetic Field Reconstruction with EFRM}

The FRM enables an efficient calculation of the normal and tangential flux densities in the air gap, assuming that the ferromagnetic material operates in a linear magnetic region. This is not the case for an interior mounted PMSM (IPMSM), which includes the saturation effect. The latter can be considered with an extended FRM, introduced by Gu et al. [14]. Unlike the traditional FRM, the EFRM considers the stator and rotor flux at the same time. The flux linkage in the air gap is decoupled into the $d$ and $q$ axis fluxes and the saturation effects in these two directions are modeled independently [19]. The $d$ axis flux density is the result of both the $I_{d}$ and PM flux, while the $q$ axis flux density originates only from $I_{q}$ and is obtained after replacing the PM with air. The flux densities in the $d$ and $q$ axis $\left(B_{d}, B_{q}\right)$ are obtained using the decoupling principle, Eq. (7) [14]:

$$
\begin{aligned}
& B_{d}=B_{d n}+B_{d t}, \quad I_{q}=0, \\
& B_{q}=B_{q n}+B_{q t}, \quad I_{d}=0, \quad P M=0,
\end{aligned}
$$

where $B_{d n}, B_{d t}, B_{q n}, B_{q t}$ represent their normal and tangential components, and the $I_{d}, I_{q}$ single current values. While neglecting the cross-coupling effect between the $d$ and $q$ axis, the normal and tangential components of the flux density distributions $\left(B_{n}, B_{t}\right)$ can be expressed as a superposition of the $d$ and $q$ axis flux densities:

$$
\begin{aligned}
& B_{n}=B_{d n}+B_{q n}, \\
& B_{t}=B_{d t}+B_{q t} .
\end{aligned}
$$

As seen from Eq. (8), the EFRM reconstructs the flux density components $B_{n}$ and $B_{t}$ by summing their $d$ and $q$ contributions, Eq. (7). These are treated separately and depend on the flux density distributions along the air-gap contour, which are pre-calculated with FEA and stored as a basis functions. The latter represent a look-up table including $B_{d n}, B_{q n}, B_{d t}, B_{q t}$ distributions along the air-gap contour at different rotor positions to take into account the spatial machine harmonics and different $I_{d}$ or $I_{q}$ current levels to consider the saturation effect. With the basis functions, the magnetic field density distribution at any rotor position with any current values lower than rated can be interpolated and rebuilt according to Eq. (8).

\subsection{Electromagnetic Forces}

The electromagnetic forces in electrical machines can be calculated with the Maxwell stress tensor (MST) method, which provides a detailed local force distribution. According to the MST, the tangential and normal force densities in the air gap can be expressed as [11] and [12]: 


$$
\begin{gathered}
f_{t}=\frac{1}{\mu_{0}}\left(B_{n} B_{t}\right), \\
f_{n}=\frac{1}{2 \mu_{0}}\left(B_{n}^{2}-B_{t}^{2}\right),
\end{gathered}
$$

where $\mu_{0}$ is the permeability of air. The cumulative tangential and radial forces acting on the tip of each stator tooth $k$ can be computed by integrating the force density components Eqs. (9) and (10) over the respective surface area $S_{k}$ :

$$
\begin{aligned}
& F_{k, t}=\int_{S_{k}} f_{t} \mathrm{~d} S_{k}, \\
& F_{k, n}=\int_{S_{k}} f_{n} \mathrm{~d} S_{k} .
\end{aligned}
$$

\subsection{Vibration Response Using Modal Decomposition}

The dynamics of an arbitrary system with $N$ degrees of freedom (DOF) is determined by the equation of motion in the time domain [20]:

$$
\boldsymbol{M} \ddot{x}(t)+\boldsymbol{D} \dot{x}(t)+\boldsymbol{K} x(t)=\boldsymbol{f}(t),
$$

where $\boldsymbol{M}, \boldsymbol{D}, \boldsymbol{K}$ are the mass, damping and stiffness matrices, $x(t)$ is the displacement, $\dot{x}(t)$ is the velocity and $\ddot{x}(t)$ is the acceleration vector. The excitation forces are denoted by $f(t)$. Using a Fourier transformation, Eq. (13) can be transformed into the frequency domain as:

$$
\left[-\omega^{2} \boldsymbol{M}+\mathrm{i} \omega \boldsymbol{D}+\boldsymbol{K}\right] \boldsymbol{X}(\omega)=\boldsymbol{F}(\omega),
$$

where $\omega$ represents the angular velocity, $i$ the imaginary unit and $\boldsymbol{X}(\omega), \boldsymbol{F}(\omega)$ the response and excitation vectors in the frequency domain, whose amplitudes are complex numbers characterizing the amplitude as well as the phase delay. Eq. (14) can be further rewritten into the response model [21]:

$$
\boldsymbol{X}(\omega)=\boldsymbol{H}(\omega) \boldsymbol{F}(\omega)
$$

where the response matrix $\boldsymbol{H}(\omega)$ contains all the combinations of the Frequency response function (FRF) between the input excitations and the output response points, as shown in Eq. (16):

$$
\boldsymbol{H}(\omega)=\left[\begin{array}{cccc}
H_{11}(\omega) & H_{12}(\omega) & \cdots & H_{1 N}(\omega) \\
H_{21}(\omega) & H_{22}(\omega) & \cdots & H_{2 N}(\omega) \\
\vdots & \vdots & \ddots & \vdots \\
H_{N 1}(\omega) & H_{N 2}(\omega) & \cdots & H_{N N}(\omega)
\end{array}\right] .
$$

The individual displacement FRF $H_{j k}(\omega)$ of a response point $j$ to an excitation at point $k$ is defined as [20]:

$$
H_{j k}(\omega)=\sum_{r=1}^{N}\left(\frac{{ }_{r} A_{j k}}{\mathrm{i} \omega-\lambda_{r}}+\frac{{ }_{r} A_{j k}^{*}}{\mathrm{i} \omega-\lambda_{r}^{*}}\right),
$$

where ${ }_{r} A_{j k}=\phi_{j r} \phi_{k r}$ is the modal constant, $\phi_{r}$ is the massnormalized eigenvector of mode $r, \lambda_{r}$ are the system eigen-values containing the angular eigenfrequencies $\omega_{r}$ and the damping ratios $\zeta_{r}$ according to:

$$
\lambda_{r}=-\zeta_{r} \omega_{r} \pm \mathrm{i} \omega_{r} \sqrt{1-\zeta_{r}^{2}} .
$$

The formulation of Eq. (17) indicates the modal decomposition, where the response equals the sum of the modes $\left(\lambda_{r},{ }_{r} A_{j k}\right)$ and their complex conjugates $\left(\lambda^{*}{ }_{r},{ }_{r} A^{*}{ }_{j k}\right)$. Finally, the vibration velocity vector $v(\omega)$, i.e., the time derivative of the displacement vector $\boldsymbol{X}(\omega)$ is expressed in the frequency domain using Eq. (15) as:

$$
\boldsymbol{v}(\omega)=\mathrm{i} \omega \cdot \boldsymbol{H}(\omega) \boldsymbol{F}(\omega) .
$$

\subsection{A-Weighted Airborne Sound Power Level}

The A-weighted airborne sound power radiated by a machine caused by the structure vibrations of its outer surface $P_{A}$ is determined with ISO/TS 7849-1 [22]:

$$
P_{A}=\sigma \rho c S \bar{v}_{a}^{2},
$$

where $\sigma$ represents the radiation efficiency, $\rho$ is the air density, $c$ is the speed of sound in the air, and $\bar{v}_{a}^{2}$ is the squared spatial average of the A-weighted vibration velocity component perpendicular to the outer surface of the machine $S$. Eq. (20) can be rewritten for the surface segments $S_{j}$ and their A-weighted normal velocity components $v_{a j}$ and thus the A-weighted sound power level $L_{W A}$ can be calculated with Eq. (21), where the reference value $P_{0}$ is $10^{-12} \mathrm{~W}$ :

$$
L_{W A}=10 \log \frac{\sigma \rho c \sum S_{j} v_{a j}^{2}}{P_{0}}
$$

\section{CASE STUDY: PWM EXCITATION OF THE IPMSM}

Section 2 shows the steps of the proposed method, which are programmed with our own code and used in the case study. This method requires a pre-calculation of the basis functions and the modal parameters, which are imported from the commercial FEA, but afterwards the effect of different PWM excitation cases can be evaluated within a few seconds. To 
prove the credibility of the proposed method, the same case study is also set up with commercial FEA tools, presented in Section 1. Then the results of both approaches are compared, and the main advantage of the proposed method, i.e., computational efficiency, is emphasized. All the calculations are made on the same desktop computer with an Intel Core i7 2.5-GHz CPU and 16 GB RAM.

The IPMSM used for the case study involves partial magnetic saturation, which can be considered with the EFRM. The main parameters of the used IPMSM are listed in Table 1. The rated value of the phase current is $3.6 \mathrm{~A}$; therefore, the basis function is obtained at different $I_{d}$ and $I_{q}$ values, within $\pm 5 \mathrm{~A}$. Details of the 2D electromagnetic and 3D mechanical models are presented in Sections 3.1 and 3.2.

Table 1. Main parameters of the used IPMSM

\begin{tabular}{lc}
\hline Outer diameter of stator & $135 \mathrm{~mm}$ \\
\hline Inner diameter of stator & $65.8 \mathrm{~mm}$ \\
\hline Number of stator slots & 12 \\
\hline Stator / rotor material & $\mathrm{M} 400-50 \mathrm{~A}$ \\
\hline Rotor diameter & $65 \mathrm{~mm}$ \\
\hline Thickness of magnet & $8 \mathrm{~mm}$ \\
\hline Magnet remanence & $0.4 \mathrm{~T}$ \\
\hline Stack length & $22 \mathrm{~mm}$ \\
\hline Airgap length & $0.4 \mathrm{~mm}$ \\
\hline Number of phases & 3 \\
\hline Number of coil turns & 116 \\
\hline Rated current & $3.6 \mathrm{~A}$ \\
\hline Rated torque & $1.7 \mathrm{Nm}$ \\
\hline Rated speed & $1500 \mathrm{RPM}$ \\
\hline
\end{tabular}

\subsection{D Electromagnetic Model}

Fig. 5 shows the 2D electromagnetic finite element (FE) model. The flux-density variation repeats every 3 stator teeth; therefore, only one-quarter of the IPMSM needs to be considered to obtain the complete flux-density distribution. As shown in Fig. 5, the rotor rotates anticlockwise and contains permanent magnets, whereas the stator is fixed and contains 3-phase windings (A, B, C). The rotor and stator generate the common rotating magnetic field.

The 2D electromagnetic FE model is used to obtain the basis functions for the EFRM and is also included in the conventional multiphysics FEA approach. The basis functions in this case study are spatially discretized with 900 points along the air-gap and obtained for 400 different rotor positions (to include the spatial machine harmonics) and 21 different current levels $I_{d}$ or $I_{q}$ from -5 A to $5 \mathrm{~A}$ (to

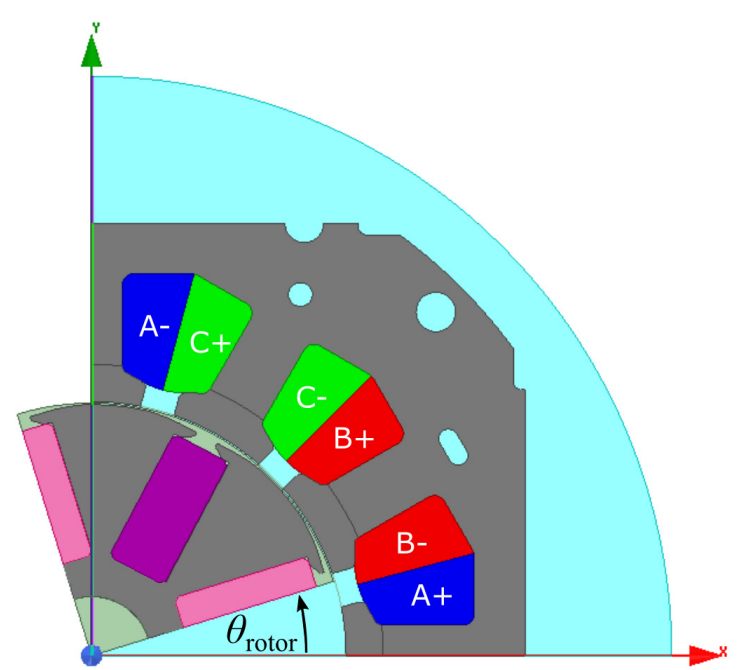

Fig. 5. 2D electromagnetic FE model

consider the saturation effect). The whole basis functions are pre-calculated in $42 \mathrm{~h}$. By using the basis functions, the flux-density distribution at any rotor position with any current value lower than the rated current can be interpolated and rebuilt with Eqs. (7) and (8). Fig. 6 shows the basis functions at rotor position $0^{\circ}$ contributed by the $d$ axis flux, which are

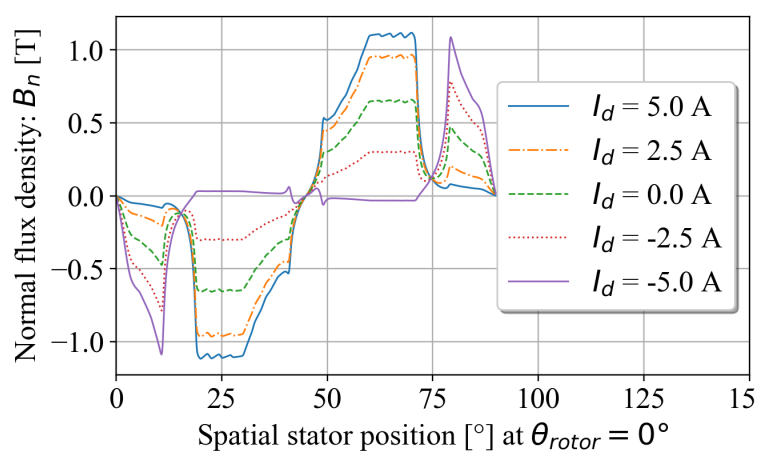

Fig. 6. Radial flux density distribution contributed by $I_{d}$

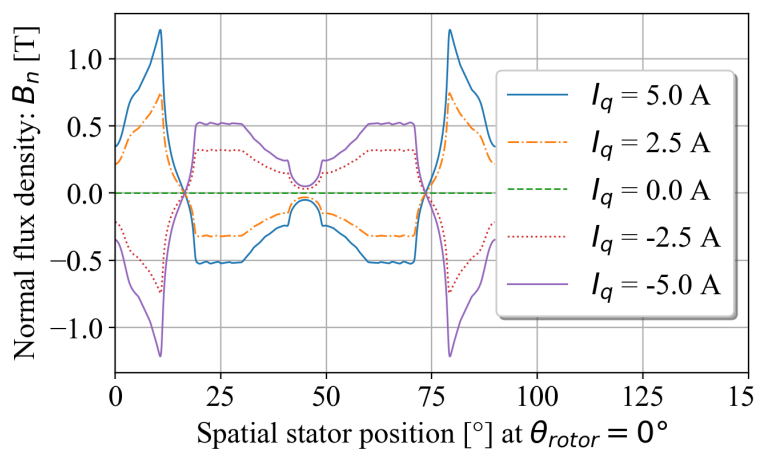

Fig. 7. Radial flux density distribution contributed by $I_{q}$ 
the result of both the PM and the different $I_{d}$ current. The curve at $I_{d}=0 \mathrm{~A}$ represents the flux-density distribution due to the PM flux only. The curves in Fig. 6 show that the PM flux density distribution increases with positive $I_{d}$ and decreases with negative $I_{d}$. The curve at $I_{d}=5 \mathrm{~A}$ indicates the saturation effect, since the increment of the flux density is degraded compared to the curve at $I_{d}=2.5 \mathrm{~A}$. Similarly, Fig. 7 shows the basis function at rotor position $0^{\circ}$ for different $I_{q}$ currents without considering the $d$ axis flux. The flux density at $I_{q}=0$ A represents the zero array, while the positive and negative $I_{q}$ values result in the opposite magnetic flux distribution.

The EFRM was validated with a co-simulation using Maxwell and Simplorer in the ANSYS commercial package. The tested case used PWM voltage excitation with the fundamental component at a frequency of $100 \mathrm{~Hz}$ and a switching frequency at $3000 \mathrm{~Hz}$, shown in Fig. 3. To compare the results in steady state at least 3 electrical cycles must be accomplished. Using a small time step $(1 \mu \mathrm{s})$ results in $3 \cdot 10000=30000$ steps, which are computed in $75 \mathrm{~h}$
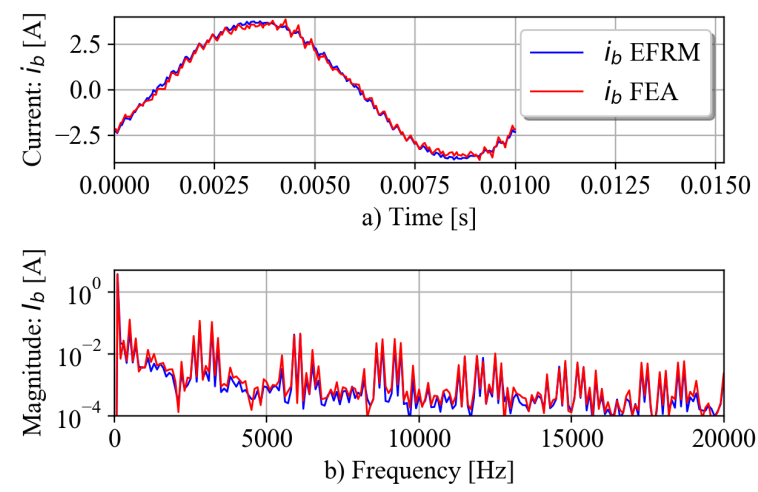

Fig. 8. Phase current at PWM excitation in a) time domain and b) in the frequency domain
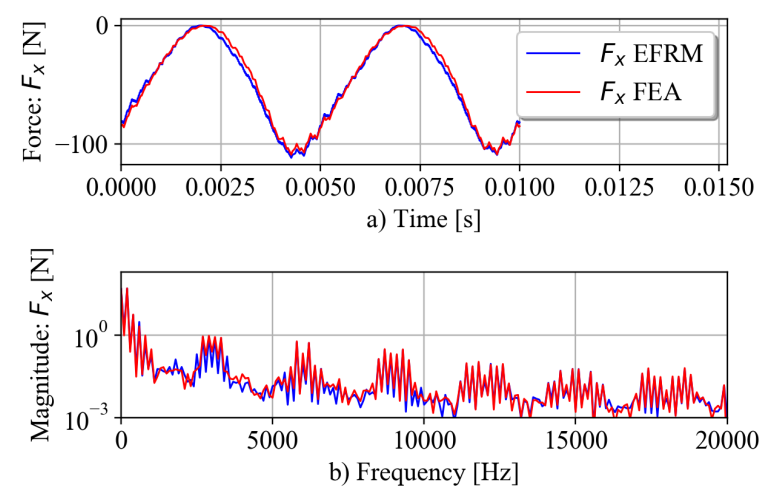

Fig. 9. Magnetic force at PWM excitation in a) time domain and b) in the frequency domain with a $2 \mathrm{D}$ electromagnetic FEA or $3 \mathrm{~s}$ with the EFRM. Figs. 8 and 9 show the resulting phase current and magnetic force in the time and frequency domains. The frequency-domain contents show that the PWM switching harmonics are also transmitted in the phase current and magnetic force. Although the EFRM is approximate, good agreement with the FEA results is seen with respect to both the time and frequency domains.

\subsection{D Mechanical Model}

The electromagnetic and mechanical models are coupled with a response model. The latter can be calculated using exported modal data from the FEA. A 3D mechanical FE model is meshed with almost 120,000 nodes, but the response model can be reduced since the only needed FRFs are between the excitation and response locations, shown in Fig. 10:

- Excitation locations

The air-gap force density around each stator-tooth tip causes the resultant net force and torque on its center. To involve both excitation type sources, every stator-tooth tip surface is halved, containing the net force on both halves. The stator has 12 teeth and therefore 24 excitation locations (index $k$ ).

- Response locations

The size of the response surfaces is defined in accordance with the well-known six-elementsper-wavelength criterion [23]. To predict the noise up to $20 \mathrm{kHz}$, the maximum response surface length should be less than one-sixth of the corresponding wavelength, which is around $2.8 \mathrm{~mm}$. As shown in Fig. 10, the model contains 1360 response locations on its outer surface (index $j$ ).

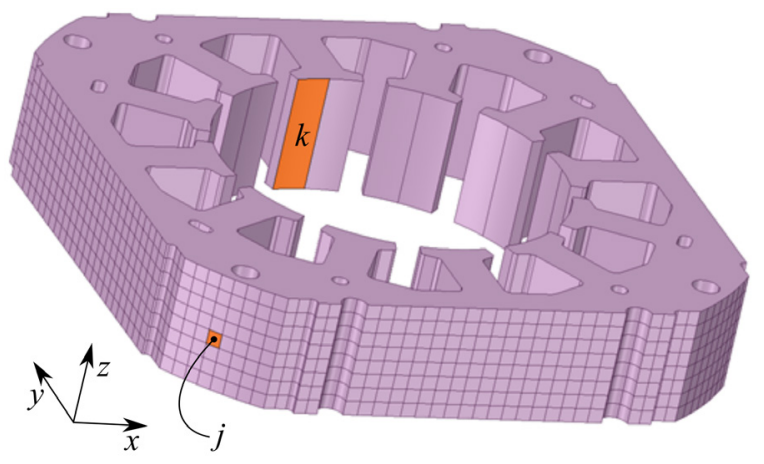

Fig. 10. 3D mechanical FE model of the stator

Using the modal decomposition, the response matrix Eq. (16) is described in terms of modal 
shapes, frequencies and damping. The modal data are imported from the commercial FEA only for the selected locations, forming the reduced response model. Thus, the length of the array for the reduced response matrix is $24 \cdot 1360 \cdot 3 \cdot 3=293760$, where the last two multipliers stands for the 3 excitation directions of each excitation location and the 3 response directions of each response location. After importing the modal data, all these displacement FRFs are calculated using Eq. (17) in 3 minutes. It should be noted that the FRFs are calculated only once and then reused for coupling different PWM excitations with structural dynamics.

Fig. 11 shows an example of the displacement FRF $H_{j x, k y}(\omega)$ of a response point $j$ in the $x$ direction to an excitation at point $k$ in the $y$ direction, which are marked in Fig. 10. The FRF curve, calculated with modal decomposition (MD), was also validated by using the Harmonic response module in the ANSYS 18.1 .

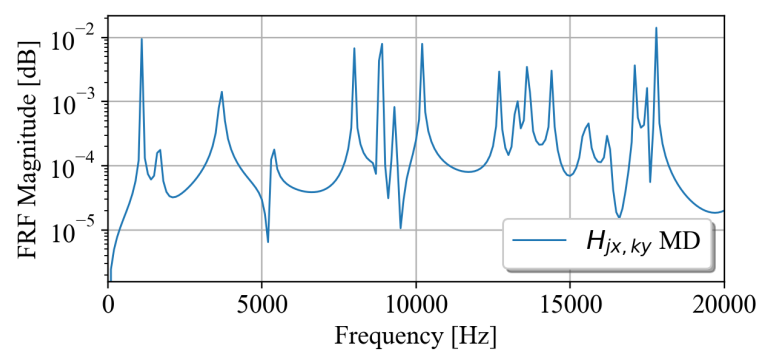

Fig. 11. FRF $H_{j x, k y}(\omega)$ from the stator-tooth tip " $k$ " to the outer subsurface " $j$ "

\subsection{Vibration Response and Sound Power Level}

Similarly as shown in Fig. 9 for single magnetic force, now magnetic forces are calculated for all 24 excitation locations in three coordinate directions $(x, y, z)$ and transformed in the frequency domain to build the force excitation vector $\boldsymbol{F}(\omega)$. By using the force excitation vector and the response matrix, Eq. (16), the vibration-velocity response vector is calculated efficiently with matrix multiplication, Eq. (19). Fig. 12 shows the vibration-velocity response in the $x$ direction for location $j$, including both, the EFRM-MD and FEA results. The frequency contents in both curves are in a good agreement, showing that the proposed method approximates the FEA results with a small error.

Further, the vibration-velocity response vector, calculated for all the response locations in three coordinate directions $(x, y, z)$, is transformed in the surfaces' normal direction, shown in Fig. 13. These vibration-velocity components represent the input for the acoustic field simulation, e.g., using the boundaryelement method [15]. However, by using the outer surface segments $S_{j}$ and the vibratory components perpendicular to them $v_{a j}$, the sound power level can also be estimated via Eq. (21). Fig. 14 shows the sound power level calculated with the FEA and with Eq. (21) using the radiation efficiency $\sigma=1$. This assumption leads to a conservative estimate of the radiated air-borne sound power, but it is valid at higher frequencies [24]. Since the article is focused on the high-frequency PWM switching noise, the presented estimation of the sound power level is acceptable.

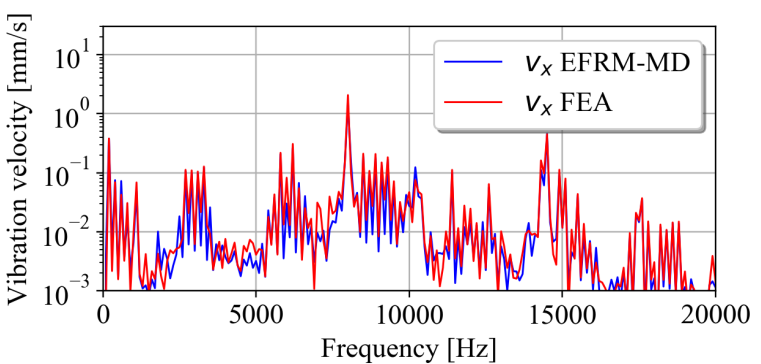

Fig. 12. Vibration response at PWM excitation in the frequency domain

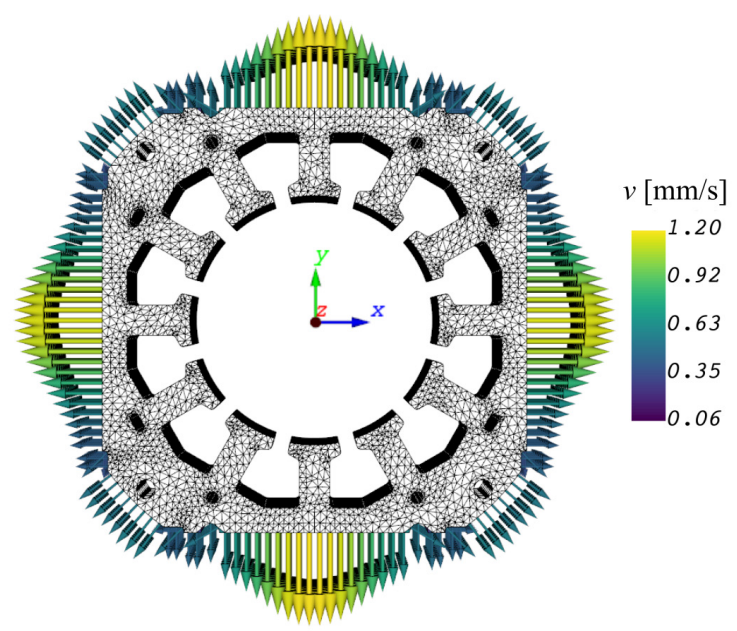

Fig. 13. Vibration response in the surfaces' normal direction

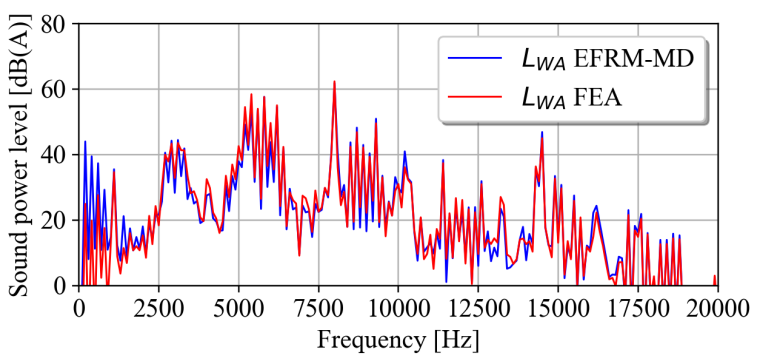

Fig. 14. Sound power level at PWM excitation in the frequency domain 


\section{PARAMETRIC STUDY AND COMPUTATIONAL EFFICIENCY}

The proposed method enables a fast sound power level estimation and therefore a parametric study can be made within a few minutes. To show the computational efficiency, an example with many different PWM excitations is used, where each contains the same fundamental component, but a different PWM carrier frequency. The included PWM excitations result in the same motor speed and torque, but contain different PWM switching harmonics, Eq. (1). The proposed approach is used for different PWM excitations to estimate the total sound power level. Its dependency on the PWM carrier frequency is shown in Fig. 15, which contains 197 different excitation cases $(400 \mathrm{~Hz}, 500 \mathrm{~Hz}, \ldots, 20000 \mathrm{~Hz})$. The excitation case, where any of the PWM switching harmonics excite the stator natural frequency, results in greater noise. However, there are also excitation cases where the PWM switching harmonics interact with the antiresonant regions in the FRF curves (Fig. 11), resulting in low total sound power levels. Fig. 15 shows that an appropriate PWM carrier frequency can decrease the total sound power level by more than $30 \mathrm{~dB}(\mathrm{~A})$.

To proove the general usage of the proposed approach, a few characteristic excitation cases were validated also with FEA, shown with black markers in Fig. 15. Mean difference in total sound power level betwen the proposed method and the FEA results is $1.5 \mathrm{~dB}(\mathrm{~A})$, while the standard deviation is $0.8 \mathrm{~dB}(\mathrm{~A})$.
Good agreement prooving that carrier-frequency selection has a great impact on structure-borne noise.

The main advantage of the proposed method is the computational efficiency. At the beginning the basis functions and the modal data must be pre-calculated using the FEA, which takes around $43 \mathrm{~h}$, but then the sound power level for any PWM excitation can be calculated in a few seconds. For example, the curve in Fig. 15 contains 197 different excitations and is calculated in 10 minutes. The same parametric study using only the conventional FEA tools in an identical computational platform would take more 600 days, i.e., $197 \cdot 75 \mathrm{~h}=14775 \mathrm{~h}$.

\section{CONCLUSION}

This article introduces a fast multiphysics numerical modeling of the structure-borne noise at PWM excitation. Firstly, the PWM excitation is used with the EFRM to obtain the electromagnetic forces on the tips of the stator teeth. Then these electromagnetic forces are coupled with the structural model to calculate the vibration-velocity response. The coupling between the electromagnetic and mechanical domains is made with the response model, which can be calculated directly in the frequency domain. Finally, the vibration-velocity response of all the outer surfaces is used to estimate the sound power level of the structure-borne noise. To prove the credibility, each step of the method is validated with the corresponding FEA.

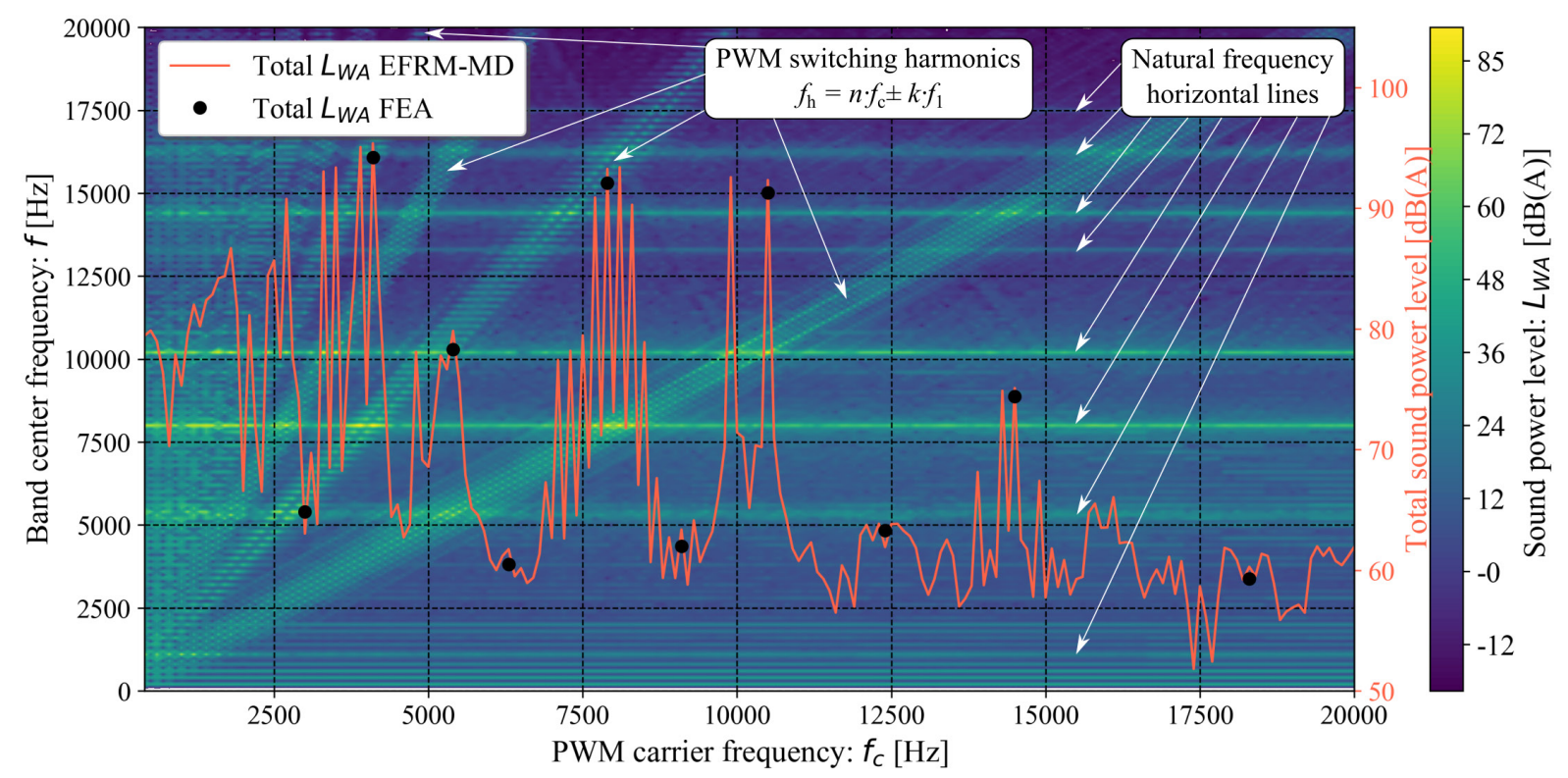

Fig. 15. Parametric study: $L_{W A}$ at different PWM carrier frequencies 
Detailed influence of the PWM carrier frequency on the structure-borne noise has been researched experimentally already before, but proposed manuscript shows how it can be estimated also numerically. Other researchers used different analytical and numerical approaches, where the first are less acurate but the second are time consuming. Instead of electromagnetic FEA an efficient EFRM can be used. EFRM was proposed in 2016, but we upgraded and validated it into the multiphysics method to calculate the structure-borne noise accurately and efficiently. If the data pre-calculation is not taken into account, the proposed method is more than $10^{4}$ times faster than a conventional multiphysics FEA and is therefore very useful for parametric noise studies at different PWM excitations.

\section{REFERENCES}

[1] Boys, J.T., Handley, P.G. (1990). Harmonic analysis of space vector modulated PWM waveforms. IEEE Proceedings $B$ Electric Power Applications, vol. 137, no. 4, p. 197-204, DOI:10.1 049/ip-b.1990.0023.

[2] Tsoumas, I.P., Tischmacher, H. (2014). Influence of the Inverter's modulation technique on the audible noise of electric motors. IEEE Transactions on Industry Applications, vol. 50, no. 1, p. 269-278, D0l:10.1109/TIA.2013.2268453.

[3] Binojkumar, A.C., Saritha, B., Narayanan, G. (2015). Acoustic noise characterization of space-vector modulated induction motor drives - an experimental approach. IEEE Transactions on Industrial Electronics, vol. 62, no. 6, p. 3362-3371, DOI:10.1109/TIE.2014.2374557.

[4] Luznar, J., Slavič, J., Boltežar, M. (2018). Experimental research on structure-borne noise at pulse-width-modulation excitation. Applied Acoustics, vol. 137, p. 33-39, D0l:10.1016/J.apac oust.2018.03.005.

[5] Le Besnerais, J., Lanfranchi, V., Hecquet, M., Brochet, P. (2010). Characterization and reduction of audible magnetic noise due to PWM supply in induction machines. IEEE Transactions on Industrial Electronics, vol. 57, no. 4, p. 12881295, DOI:10.1109/TIE.2009.2029529.

[6] Islam, R., Husain, I. (2010). Analytical model for predicting noise and vibration in permanent-magnet synchronous motors. IEEE Transactions on Industry Applications, vol. 46, no. 6, p. 2346-2354, DOI:10.1109/TIA.2010.2070473.

[7] Le Besnerais, J., Lanfranchi, V., Hecquet, M., Brochet, P., Friedrich, G. (2010). Prediction of audible magnetic noise radiated by adjustable-speed drive induction machines. IEEE Transactions on Industry Applications, vol. 46, no. 4, p. 13671373, DOI:10.1109/TIA.2010.2049624.

[8] Lin, F., Zuo, S., Deng, W., Wu, S. (2016). Modeling and analysis of electromagnetic force, vibration, and noise in permanentmagnet synchronous motor considering current harmonics. IEEE Transactions on Industrial Electronics, vol. 63, no. 12, p. 7455-7466, DOI:10.1109/TIE.2016.2593683.
[9] Pellerey, P., Lanfranchi, V., Friedrich, G. (2012). Coupled numerical simulation between electromagnetic and structural models. Influence of the supply harmonics for synchronous machine vibrations. IEEE Transactions on Magnetics, vol. 48, no. 2, p. 983-986, D0I:10.1109/TMAG.2011.2175714.

[10] Druesne, F., Hallal, J., Lardeur, P., Lanfranchi, V. (2016). Modal stability procedure applied to variability in vibration from electromagnetic origin for an electric motor. Finite Elements in Analysis and Design, vol. 122, p. 61-74, D0l:10.1016/j. finel.2016.09.004.

[11] Zhu, W., Fahimi, B., Pekarek, S. (2006). A field reconstruction method for optimal excitation of permanent magnet synchronous machines. IEEE Transactions on Energy Conversion, vol. 21, no. 2, p. 305-313, Dol:10.1109/ TEC.2005.859979.

[12] Sutthiphornsombat, B., Khoobroo, A., Fahimi, B. (2010). Mitigation of acoustic noise and vibration in permanent magnet synchronous machines drive using field reconstruction method. IEEE Vehicle Power and Propulsion Conference, $p$. 1-5, DOl:10.1109/VPPC.2010.5728997.

[13] Torregrossa, D., Fahimi, B., Peyraut, F., Miraoui, A. (2012). Fast computation of electromagnetic vibrations in electrical machines via field reconstruction method and knowledge of mechanical impulse response. IEEE Transactions on Industrial Electronics, vol. 59, no. 2, p. 839-847, Dol:10.1109/ TIE.2011.2143375.

[14] Gu, L., Moallem, M., Bostanci, E., Wang, S., Devendra, P. (2016). Extended field reconstruction method for modeling of interior permanent magnet synchronous machines. IEEE Transportation Electrification Conference and Expo (ITEC), p. 1-6, D0I:10.1109/ITEC.2016.7520252.

[15] Furlan, M., Černigoj, A., Boltežar, M. (2003). A coupled electromagnetic-mechanical-acoustic model of a DC electric motor. COMPEL-The International Journal for Computation and Mathematics in Electrical and Electronic Engineering, vol. 22, no. 4, p. 1155-1165, Dol:10.1108/03321640310483075.

[16] Torregrossa, D., Paire, D., Peyraut, F., Fahimi, B., Miraoui, A. (2012). Active mitigation of electromagnetic vibration radiated by PMSM in fractional-horsepower drives by optimal choice of the carrier frequency. IEEE Transactions on Industrial Electronics, vol. 59, no. 3, p. 1346-1354, Dol:10.1109/ TIE.2010.2081961.

[17] Neugebauer, H. (2012). Parameter Identification of a Permanent Magnet Synchronous Motor. MSc thesis, Chalmers University of Technology, Gothenburg.

[18] Khoobroo, A., Fahimi, B., Pekarek, S.D. (2008). A new field reconstruction method for permanent magnet synchronous machines. 34th Annual Conference of IEEE Industrial Electronics, p. 2009-2013, D0l:10.1109/ IECON.2008.4758265.

[19] Gu, L. (2016). Modeling of permanent Magnet Machines Using Field Reconstruction Method. PhD thesis, The University of Texas, Dallas.

[20] Maia, N.M.M., Silva, J.M.M. (1997). Theoretical and experimental Modal Analysis. Research Studies Press, Taunton.

[21] Ewins, D.J. (1984). Modal Testing: Theory and Practice. John Wiley \& Sons, Ontario. 
[22] ISO/TS 7849-1 (2009). Acoustics: Determination of Airborne Sound Power Levels Emitted by Machinery Using Vibration Measurement. International Organization for Standardization, Geneva.
[23] Marburg, S. (2002). Six boundary elements per wavelength: Is that enough?. Journal of Computational Acoustics, vol. 10, no. 1, p. 25-51, DOI:10.1142/S0218396X02001401.

[24] Gieras, J.F., Wang, C., Lai, J.C. (2006). Noise of Polyphase Electric Motors. CRC Press, Boca Raton. 